\title{
Signatures of Quark-Gluon Plasma Phase Transition in High-Energy Nuclear Collisions
}

\author{
Cheuk-Yin Wong ${ }^{\text {a* }}$

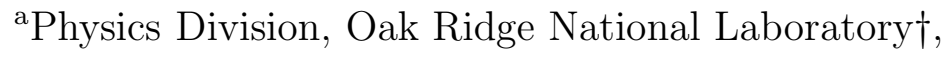 \\ Oak Ridge, TN 37831, U.S.A.
}

In high-energy nuclear collisions, the new phase of the quark-gluon plasma is indicated by an anomalous increase in pressure, an excess of direct photon production, an excess of strangeness production, and an anomalous $J / \psi$ suppression. We review these signatures and discuss how recent high-energy heavy-ion experiments at CERN are consistent with the production of the quark-gluon plasma in high-energy $\mathrm{Pb}+\mathrm{Pb}$ collisions.

\section{INTRODUCTION}

A recent news release from CERN stated that a new state of matter, the quark-gluon plasma (QGP), was created by high-energy heavy-ion collisions at CERN. The evidence for such an observation was summarized by Heinz and Jacobs [1]. The news release has generated a great deal of excitement. It is useful to review and re-examine this evidence from various perspectives to see whether such an excitement is justified or not.

In this review and re-examination, we shall discuss the nature of the order of the hadronQGP phase transition, the dynamics of how such a transition takes place in high-energy heavy-ion collisions, and the signatures for the phase transition. Prominent signatures include the large pressure in the quark-gluon plasma phase which leads to an anomalous increase in the freeze-out volume, the emission of direct photons when the matter is in the quark-gluon plasma phase [2], the strong enhancement of strangeness [3], and the suppression of $J / \psi$ production [4]. Other evidence can also be found by mapping out the phase boundary in the phase diagram from the distribution and the yield of hadron products, which will be reported by Braun-Munzinger in these Proceedings [5]. Our reassessment of these signatures provides additional support to indicate that, in line with the summary of Heinz and Jacobs [1], the experimental data appear to be consistent with the occurrence of the quark-gluon plasma.

\section{THE QUARK-GLUON PLASMA PHASE TRANSITION}

A phase transition is classified as a first- or second-order transition depending on how its free energy varies with the order parameter. The phase transition is first order if the free energy as a function of the order parameter exhibits two local minima at different values

\footnotetext{
${ }^{* \dagger}$ Managed by UT-Battelle, LLC for the U.S. Department of Energy under Contract DE-AC0500OR22725.
} 
of the order parameter, and the order parameter of the lowest minimum changes from one local minimum to the other discontinuously as the temperature passes the critical temperature $T_{\text {crit }}$ as shown schematically in Fig. 1a. A phase transition is second order if the free energy as a function of the order parameter exhibits a single minimum, and the location for the minimum changes continuously for different values of the order parameter as the temperature passes the critical temperature $T_{\text {crit }}$ (Fig. 1b). (See also Fig. 11.3 and 11.4 of Ref. [6].)

The order parameter for strongly interacting matter is the Wilson line parameter, which is related to the interaction energy $V$ when an isolated quark is placed in the medium. It is proportional to $e^{-V t}$ for a Wilson line of temporal length $t$. In the hadron phase, because of the linear interaction between a quark and an antiquark, the interaction energy $V$ is infinite when an isolated quark is placed in the medium and the Wilson line parameter is zero. The Wilson line parameter is non-zero in the deconfined phase.

Lattice gauge calculations show that the transition is first order for a quarkgluon plasma with two flavors, but changes to a second-order phase transition for three flavors with the physical mass of the strange quark [7]. These theoretical predictions need to confront experimental data to deduce the nature of the phase transition.

How does one visualize the difference between the hadron matter and the quark-gluon plasma? One can envisage a spatial distribution of the link
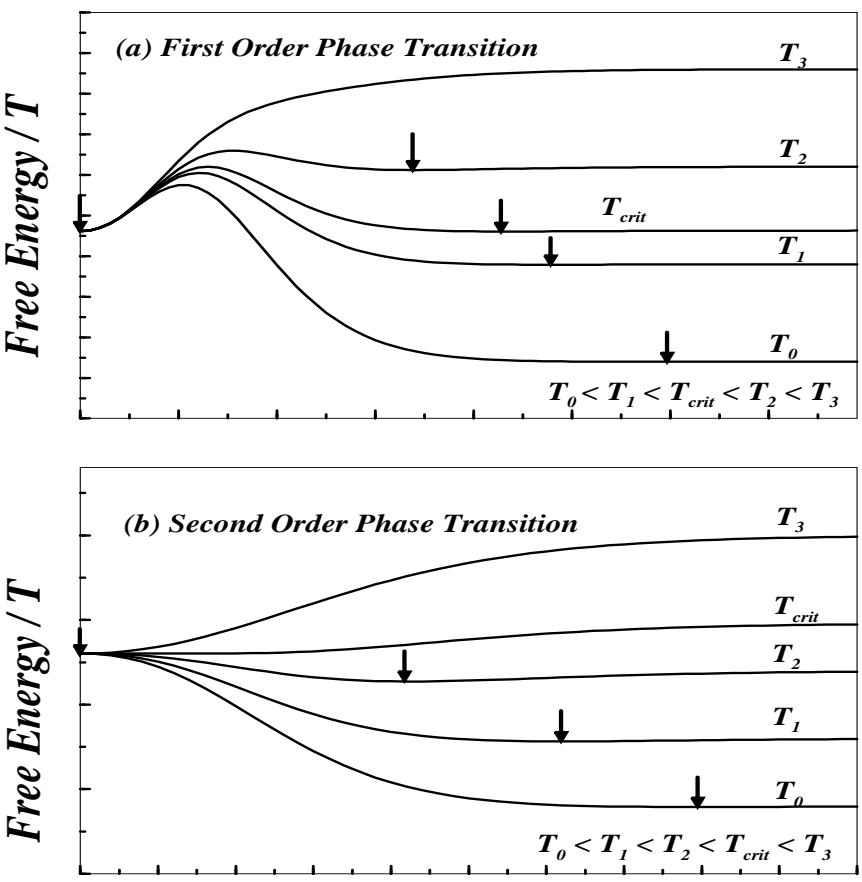

\section{Order Parameter}

Fig. 1 The free energy divided by the temperature as a function of the order parameter for different temperature $T$. Fig. $1 a$ shows a first-order phase transition, and Fig. $1 b$ shows a secondorder phase transition. An arrow indicates the location of a local minimum. variables which contain the gluon degrees of freedom and the generators of SU(3). The link variables in strongly interacting matter are the analogue of spins in a spin gas. The hadron phase is the low-temperature phase in which neighboring link variables (in a plaquette) are all correlated in order to reside in the lowest energy state. The correlation can be considered roughly as some generalized 'alignment' of the link variables, in analogy with the alignment of spins in the spin lattice gas. In such an 'aligned' state of the link variables in all regions of space, the interaction between a quark and an antiquark at large distances becomes a linear function of the distance, which leads to the confinement of quarks and gluons. In contrast, in the high-temperature quark-gluon plasma phase, the temperature is so high that the tendency to align the link variables due to the QCD interaction is overwhelmed by the tendency to disalign the link variables due to the thermal 
fluctuation. The link variables are no longer aligned. The interaction between a quark and an antiquark above the critical temperature at large distances is no longer governed by the linear interaction, and the quarks and gluons become deconfined.

The deconfinement phase is further accompanied by the restoration of chiral symmetry [8] in which the light quarks in the hadron phase are restored to their nearly massless current quark mass values. Thus, the phase transition from hadron matter to the quarkgluon plasma can be described as a change of the link variables from an ordered aligned state to a state of disorientation, accompanied by an abundant production of nearly massless quark pairs and additional gluons. As the link variables representing the gluons are now free to orient randomly and the nearly massless light quark pairs are copiously produced, the degree of freedom is greatly enlarged and the energy density of the deconfined phase is much greater as a result.

\section{HEAVY-ION COLLISIONS TO PRODUCE THE PHASE TRANSITION}

High-energy heavy-ion collisions can be used to produce a phase transition from the hadron matter to the quark-gluon plasma. Because of the Lorentz contraction, the nucleon-nucleon collisions in a nucleus-nucleus collision occur at about the same time and at nearly the same spatial proximity. As a consequence, a region of very high energy density can be created.

The dynamics of such a nucleus-nucleus collision can be best described in the nucleonnucleon center-of-mass system. For a given impact parameter, we can divide the transverse area of the colliding nuclei into rows with an area of a nucleon-nucleon cross section $\sigma_{i n}$. Within each row, each projectile nucleon will collide with each target nucleon. By assuming straight-line spacetime trajectories, the space-time diagram of these nucleons is shown in Fig. 2.

We can focus our attention at one spa-

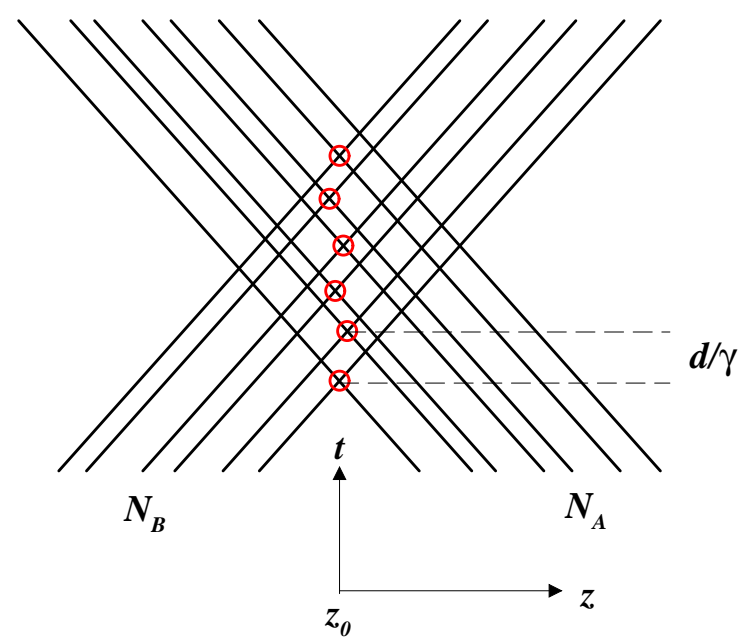

Fig. 2. The space-time diagram of a row of $N_{B}$ projectile nucleons colliding with a row of $N_{A}$ target nucleons.

tial point at $z_{0}$ in this row. A nucleon-nucleon collision at this point (represented by an open circle in Fig. 2) will lead to the deposition of energy about that point. At a proper time of about $1 \mathrm{fm} / \mathrm{c}$, the deposited energy will materialize as field quanta of matter in the form of hadrons if the matter is favored to be in the hadronic state. The field quanta will be quarks and gluons if the matter is favored to be in the quark-gluon plasma state.

There are many other nucleon-nucleon collisions which take place sequentially at $z_{0}$ at a time interval of $\sim d / \gamma$, as represented by the open circles in Fig. 2. Here, $d \sim 2.5 \mathrm{fm}$ is the average nucleon-nucleon spacing in nuclear matter at rest and $\gamma$ is the relativistic factor for the motion of the nuclei in the nucleon-nucleon center-of-mass system. Each later nucleon-nucleon collision at the same collision point deposits additional energy. The local energy density increases as a function of time and is approximately proportional to 
the number of nucleon-nucleon collisions $N$ occurring at that point:

$$
\epsilon \sim N \frac{d n}{d y} \frac{m_{t}}{\sigma_{i n} d / \gamma},
$$

where $d n / d y$ is the average multiplicity per unit of rapidity at $y_{C M}=0$ for a nucleon-nucleon collision, and $m_{t} \sim 0.35 \mathrm{GeV}$ is the transverse mass of a produced pion. For collisions at $158 \mathrm{~A} \mathrm{GeV}$ per nucleon, the energy density deposited per nucleon-nucleon collision as given by Eq. (1) is approximately $N \times 1 \mathrm{GeV} / \mathrm{fm}^{3}$.

In actual nucleus-nucleus collisions, nucleons lose energy as they collide and the multiplicity distribution in later collisions will be slightly lower than those from earlier collisions. Equation (1), which is defined in terms of an average multiplicity value, is a simplifying, but useful, relation which gives an approximate estimate of the local energy density.

As the size of the colliding nuclei increases, the energy density deposited at a collision point in a central collision will increase. When the number of nucleon-nucleon collisions at a spatial point exceeds a critical number $N_{c}$, the local energy density will increase beyond the critical energy density $\epsilon_{c}$ for transition to the quark-gluon plasma phase [9]. Then, the state of lowest free energy becomes the QGP phase with field quanta of quarks, antiquarks, and gluons. If the number of nucleon-nucleon collisions at a point is lower than the critical collision number $N_{c}$, it will not reach the critical energy density and the field quanta will be hadrons consisting mostly of pions.

\section{ANOMALOUS FREEZE-OUT VOLUME}

In a first-order phase transition, the energy density and pressure change abruptly from the hadron phase to the quark-gluon plasma phase. Consequently, the pressure changes abruptly as a function of the local collision number when the collision number passes the critical value, $N_{c}$.

The pressure in the quark-gluon plasma is given approximately by $P_{\mathrm{QGP}} \sim 37 \pi^{2} T^{4} / 90$ which is much greater than the pressure of a pion gas, $P_{\text {pion }}=6 \pi^{2} T^{4} / 90$. Thus, the presence of a quark-gluon plasma is characterized by a region of very high pressure. The large pressure of the quark-gluon plasma can be detected by a large freeze-out volume.

We would like to examine the relation between the initial pressure and the freeze-out volume in Bjorken hydrodynamics [10]. The matter produced in high-energy heavy-ion collisions is subject to a strong longitudinal expansion. We consider a plateau distribution of matter within rapidity range $|y|<y_{0}$ and longitudinal coordinates $|z|<\left|z_{0}\right|$. The initial proper time is then $\tau_{0}=\left|z_{0}\right| / \sinh y_{0}$. The solution for the pressure $P$ in Bjorken hydrodynamics is

$$
P(\tau, y)=P(\tau) \theta\left(y_{0}-|y|\right),
$$

where

$$
\frac{P(\tau)}{P\left(\tau_{0}\right)}=\left(\frac{\tau_{0}}{\tau}\right)^{4 / 3} .
$$

(See Eq. (13.17b) of Ref. [6].) At the proper time $\tau$, the boundary of the matter is expanded up to $z_{\tau}= \pm \tau \sinh y_{0}$, and thus the ratio of $\left|z_{\tau}\right| /\left|z_{0}\right|$ is

$$
\frac{\left|z_{\tau}\right|}{\left|z_{0}\right|}=\left(\frac{P\left(\tau_{0}\right)}{P(\tau)}\right)^{3 / 4} \text {. }
$$


As the matter expands, there will be a pressure $P\left(\tau_{f}\right)$, the freeze-out pressure, at which particles will no longer interact and will exhibit freeze-out characteristics. Because the volume of matter $V$ is proportional to $\left|z_{\tau}\right|$, the ratio of the freeze-out volume $V\left(\tau_{f}\right)$ at $\tau_{f}$ to the initial volume $V\left(\tau_{0}\right)$ is

$$
\frac{V\left(\tau_{f}\right)}{V\left(\tau_{0}\right)}=\left(\frac{P\left(\tau_{0}\right)}{P\left(\tau_{f}\right)}\right)^{3 / 4}
$$

Hence, the freeze-out volume $V\left(\tau_{f}\right)$ varies with the initial pressure as $\left[P\left(\tau_{0}\right)\right]^{3 / 4}$.

Equation (5) is applicable to hadron matter. It is also applicable approximately to the QGP undergoing strong longitudinal expansion, for which the temperature of the QGP is lowered so rapidly that the expansion drives the system below the critical temperature. When the energy density and pressure of the supercooled quark-gluon plasma drop down to the same level as those of the hadron matter, spontaneous transition to hadron matter will take place at proper time $\tau_{H}$ resulting in hadron matter of volume $V\left(\tau_{H}\right)$. The hadron matter then undergoes further longitudinal expansion to reach the freeze-out volume $V\left(\tau_{f}\right)$ at proper time $\tau_{f}$. For the quark-gluon plasma subject to a strong longitudinal expansion, we have

$$
\frac{V\left(\tau_{f}\right)}{V\left(\tau_{0}\right)}=\frac{V\left(\tau_{f}\right)}{V\left(\tau_{H}\right)} \frac{V\left(\tau_{H}\right)}{V\left(\tau_{0}\right)} \sim\left(\frac{P\left(\tau_{H}\right)}{P\left(\tau_{f}\right)}\right)^{3 / 4}\left(\frac{P\left(\tau_{0}\right)}{P\left(\tau_{H}\right)}\right)^{3 / 4}=\left(\frac{P\left(\tau_{0}\right)}{P\left(\tau_{f}\right)}\right)^{3 / 4} .
$$

The freeze-out volume varies approximately with the initial QGP pressure as $\left[P\left(\tau_{0}\right)\right]^{3 / 4}$.

Based on the above, the large pressure of the quark-gluon plasma will lead to a large freeze-out volume and a first-order phase transition will be indicated by an anomalous increase of the freeze-out volume due to the increase in the pressure in the QGP phase. We expect that the anomalous increase in the freeze-out volume should occur when the average number of nucleon-nucleon collisions passes through the critical value $N_{c}$. The average nucleon-nucleon collision number in a collision is a function of centrality or multiplicity, so the freeze-out volume should increase anomalously as a function of centrality or multiplicity in a first-order phase transition. In contrast, the increase should be much more gradual in a second-order phase transition.

In actual heavy-ion collisions, the freeze-out volume also increases with centrality because of the increase in the volume of the overlapping region. Consequently, there will be the systematics of such a normal increase of the freeze-out volume, as one goes from the peripheral region to the central region. However, the increase in the freeze-out volume due to the large pressure of the quark-gluon plasma is an anomalous addition to these systematics. By looking at the systematics of the freeze-out volume as a function of centrality or multiplicity, one should be able to separate out these two components: the normal component due to the increase in the volume of the collision region for peripheral and semi-central collisions, and an anomalous part due to the high pressure of the quark-gluon plasma which becomes important for central collisions.

Recently the NA44 Collaboration [11] observed that the freeze-out volume increased anomalously from semi-central collisions to central collisions, for $\mathrm{Pb}+\mathrm{Pb}$ collisions at 158A GeV (Fig. 3). This interesting observation by the NA44 Collaboration may be evidence for the quark-gluon plasma. In semi-central collisions, the producded mat- 
ter are likely to be hadron matter. Therefore, the expansion and the freeze-out volume is governed mainly by the expanding hadron matter with $P\left(\tau_{0}\right) \sim P_{\text {pion }}$ and $V_{\text {freeze-out }} \propto P_{\text {pion }}^{3 / 4}$. For central collisions, if quark-gluon plasma is produced, the expansion and cooling for these central collisions is governed essentially by the initial pressure of the quark-gluon plasma, $P\left(\tau_{0}\right) \sim P_{Q G P}$, which is a very high pressure, and $V_{\text {freeze-out }} \propto P_{\mathrm{QGP}}^{3 / 4}$. Therefore, this expansion will lead to an anomalous increase in the freeze-out volume for central collisions, much greater than what one would expect from the systematics for semi-central collisions. The rapid increases of the freeze-out volume as a function of multiplicity, as observed by the NA44 Collaboration, may indicate that the hadron-QGP phase transition is a first-order phase transition. Further investigations are needed to shed more light on this interesting observation of the NA44 Collaboration.

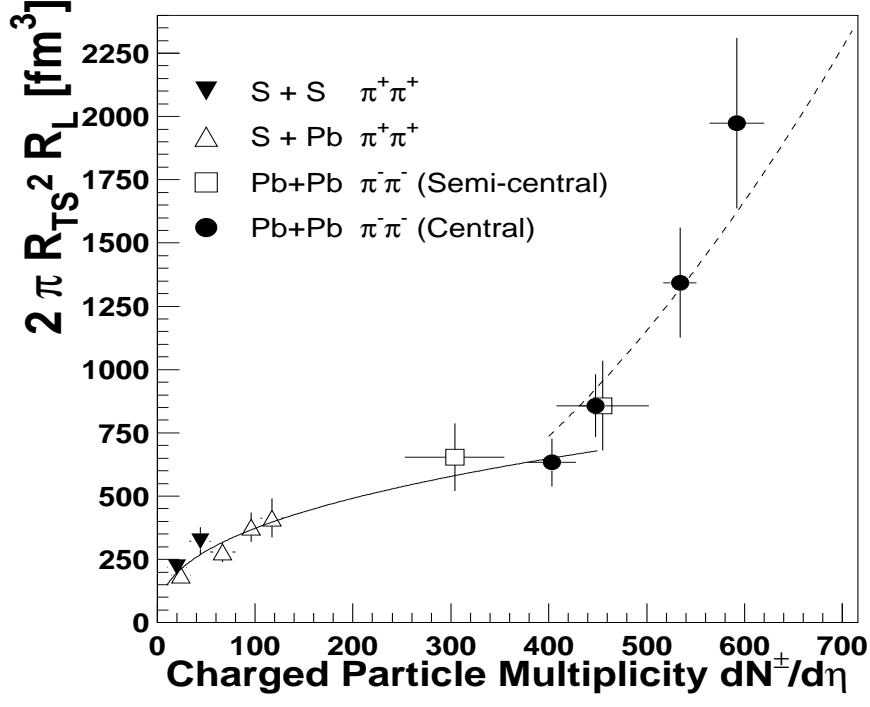

Fig. 3. The NA44 data of the freeze-out volume as a function of $d N^{ \pm} / d \eta$ [11]. The curves joining the data points are parametrizations in the form of $\left(d N^{ \pm} / d \eta\right)^{\alpha}$ from the NA44 Collaboration.

\section{DIRECT PHOTON PRODUCTION}

As the quark-gluon plasma expands, its energy density and temperature decreases. At the point when its temperature decreases below the critical temperature for hadron-QGP phase transition, matter will undergo a phase transition from the quark-gluon plasma to the hadron phase.

During the time when the matter is in the quark-gluon plasma phase, it will emit particles. Photons arising from the electromagnetic interactions of the constituents of the plasma will provide information on the properties of the plasma at the time of their emission. Since photons are hardly absorbed by the medium, they form a relatively 'clean' probe to study the state of the quark-gluon plasma. The presence of these photons in high-energy heavy-ion collisions can also possibly provide evidence for the production of the quark-gluon plasma [2, 12, 13].

Photons are also produced by many other processes in heavy-ion reactions. They can come from the decay of $\pi^{0}$ and $\eta^{0}$. As $\pi^{0}$ particles are copiously produced in strong interactions between nucleons, photons coming from the decay of $\pi^{0}$ are much more abundant than photons produced by electromagnetic interactions of the constituents of the quarkgluon plasma. The photons from the decay of $\pi^{0}$ and $\eta^{0}$ can be subtracted out by making a direct measurement of their yields, obtained by combining pairs of photons. Because of 
the large number of $\pi^{0}$ produced, this subtraction is a laborious task, but much progress has been made to provide meaningful results after the subtraction of the photons from the $\pi^{0}$ and $\eta^{0}$ backgrounds [14. Photon measurements obtained after the subtraction of the photons from meson decays are conveniently called measurements of "direct photons".

Direct photons are produced from the interaction of matter in the QGP phase, a mixed QGP and hadron phase, a pure hadron gas, and hard QCD processes. Different processes give rise to photons in different momentum regions. One may wish to go to the region of photon transverse momentum $p_{T}>2 \mathrm{GeV} / \mathrm{c}$ to minimize the contributions from hadrons. If a hot quark-gluon plasma is formed initially, clear signals of photons from the plasma could be visible by examining photons with $p_{T}$ in the range $2-3 \mathrm{GeV} / \mathrm{c}$ [12, [3]. On the other hand, photons in this region of transverse momenta are also produced by the collision of partons of the projectile nucleons with partons of the target nucleons. Such a contribution must be subtracted in order to infer the net photons from the quark-gluon plasma sources.

Recently, the WA98 Collaboration has measured the photon spectrum for central $\mathrm{Pb}+\mathrm{Pb}$ collisions at $158 \mathrm{~A}$ $\mathrm{GeV}$ [14. The photon spectrum from the hard scattering of the nucleons

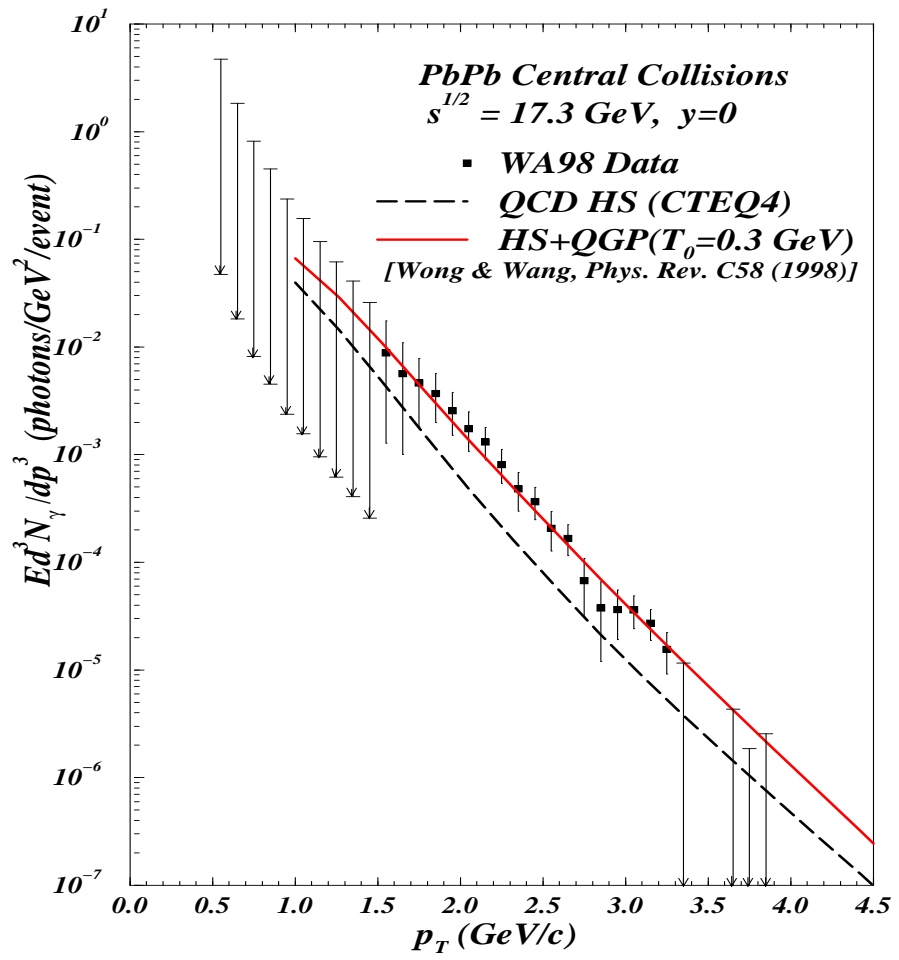

Fig. 4. The spectrum of direct photons. The data are from the WA98 Collaboration [14], and the curves are from Ref. [15].

has been calculated previously by taking into account the next-to-leading order contributions and the intrinsic transverse momentum of partons. The results [15] scaled to the $\mathrm{Pb}+\mathrm{Pb}$ collisions are shown as the dashed curve in Fig. 4. One finds that the WA98 experimental data is in excess of the contributions from the hard scattering of the nucleons.

We can calculate the total photon spectrum including the contributions from the nucleon hard scattering and the quark-gluon plasma. If we assume a quark-gluon plasma with a transverse area of the overlapping area appropriate for the corresponding impact parameter and an initial plasma formation time at $\tau=1 \mathrm{fm} / \mathrm{c}$, with a temperature of 300 $\mathrm{MeV}$ cooling to a critical temperature of $200 \mathrm{MeV}$, we obtain the photon spectrum in Fig. 9 of Ref. [15] shown here as the solid curve. The good agreement of the model with the WA98 data provides additional support for the production of the quark-gluon plasma in high-energy central $\mathrm{Pb}+\mathrm{Pb}$ collisions. 


\section{Strangeness enhancement}

The strangeness content is enhanced in hadron matter as the temperature increases, but the strangeness is enhanced to an even greater extent in a quark-gluon plasma. The greater strangeness enhancement arises from a higher temperature in the quark-gluon plasma and from its lower effective light quark masses because of the restoration of chiral symmetry. As the strangeness content is greatly enhanced, the probability for the production of multi-strange hyperons will also be greatly enhanced in a quark-gluon plasma [3].

In a recent measurement of the WA97 Collaboration [16], the production of multistrange hyperons is found to be substantially enhanced. In particular, the production of $\Omega^{-}+\overline{\Omega^{+}}$in $\mathrm{Pb}+\mathrm{Pb}$ collisions at $158 \mathrm{~A} \mathrm{GeV}$ is enhanced by up to a factor of 15 relative to that of $\mathrm{p}+\mathrm{Be}$. A more detailed description of the strangeness enhancement will be presented by Odyniec in these Proceedings [17].

Multi-strange hyperons can also be produced by secondary collisions of hadrons. It is known that the collision of the produced pions with nucleons leads to the enhancement of kaons and $\Lambda$ particles. Repeated collisions of kaons in the medium with a $\Lambda$ particle can raise the strangeness of the hyperon by one or more units. The enhancement of hyperons by these secondary collisions increases with the increase in the size of the colliding nuclei, and it is important to take this increase into account. Recent comparison of the hyperon yields as obtained by theoretical RQMD cascade model calculations [18] shows that the RQMD calculations can reproduce the yields of strange particles with one or two units of strangeness, but it underpredicts the yield of $\Omega^{-}$by a factor of 3 , and underpredicts the yield of $\overline{\Omega^{+}}$by about $40 \%$ [19].

The discrepancies of the yields of the $\Omega$ hyperons with the RQMD cascade model may be additional evidence for the production of the quark-gluon plasma in $\mathrm{Pb}+\mathrm{Pb}$ collisions. This may be the case, but such a conclusion relies heavily on the assumed input of many strangeness-raising cross sections, for which no experimental data are available. The evidence will be substantially strengthened if the strangeness-raising cross sections can be better determined by a reliable theoretical model, such as the quark-interchange model of Barnes and Swanson [20]. As emphasized by Ko [21], the strangeness-raising cross section for the reaction such as $K^{0}+\Xi^{-} \rightarrow \pi^{0}+\Omega^{-}$can proceed through the interchange of a strange quark from $K$ to $\Xi$, which need not be OZI-suppressed as in the $\pi+N \rightarrow K+\Lambda$ reaction, where an intermediate strange quark pair is produced. Future theoretical evaluation of these strangeness-raising cross sections will be of great interest in clarifying the origin of the enhancement of the $\Omega$ hyperons in $\mathrm{Pb}+\mathrm{Pb}$ collisions.

\section{7. $J / \psi$ Suppression}

In a quark-gluon plasma the screening of the charm quark and antiquark will make the $J / \psi$ unbound, and its production will be suppressed. The occurrence of $J / \psi$ suppression has been suggested as a signature for the quark-gluon plasma [4]. The experimental observation by the NA50 Collaboration [22 24] of an anomalous $J / \psi$ suppression in $\mathrm{Pb}+\mathrm{Pb}$ collisions has led to the suggestion that this anomalous suppression arises from the production of the quark-gluon plasma [9,25,26]. The phenomenon of anomalous $J / \psi$ suppression has also been studied by many authors [27.

The production of $J / \psi$ is suppressed not only by the quark-gluon plasma but also by 
the collision of the $J / \psi$ (or its precursor) with nucleons and produced particles. The absorption of $J / \psi$ by these particles was considered in a simple analytical model 9]. One divides the transverse area into rows of nucleons of cross section $\sigma_{i n}$. The projectile nucleons in each row collide with the target nucleons within the same row. One assumes simple straight-line space-time trajectories for the colliding nucleons as in Fig. 2. In this space-time diagram, each nucleon-nucleon collision is a possible source of $J / \psi$ production and also a source of produced particles which will absorb $J / \psi$. By parametrizing the dissociation cross section of $J / \psi$ in its collision with nucleons or produced particles, the $J / \psi$ production cross section in a nucleus-nucleus collision can be evaluated.

It was found that the simple model of $J / \psi$ absorption by nucleons and produced particles can explain the $p+\mathrm{A}, \mathrm{O}+\mathrm{A}$, and $\mathrm{S}+\mathrm{U}$ data, but not the $\mathrm{Pb}+\mathrm{Pb}$ data. The deviation of $\mathrm{Pb}+\mathrm{Pb}$ data from the theoretical extrapolations suggests that there is a transition to a new phase of strong absorption, the quark-gluon plasma, which sets in when the local energy density exceeds a certain threshold. As we remarked before, the energy density at a particular spatial point is approximately proportional to the number of nucleon-nucleon collisions which have taken place at that point. We postulate that the matter at a point makes a transition to the new phase of strong $J / \psi$ absorption if there have been $N_{c}$ or more nucleon-nucleon collisions at that point.

We assume that the deconfinement temperature is greater than the dissociation temperatures for the dissociation of both $\chi$ and $J / \psi$. Then, $N_{c}=4$ gives very good agreement with the $\mathrm{pA}, \mathrm{O}+\mathrm{A}, \mathrm{S}+\mathrm{U}$, and $\mathrm{Pb}+\mathrm{Pb}$ data. The critical nucleon-nucleon collision number, $N_{c}=4$, corresponds to a critical energy density of $\epsilon_{c}=4.2 \mathrm{GeV} / \mathrm{fm}^{3}$. The results for the ratio of the $J / \psi$ cross section to the Drell-Yan cross section is shown as the solid curve in Fig. 5. The good agreement of this critical energy density model [9] with the WA50 data provides evidence for the production of the quark-gluon plasma in central high-energy $\mathrm{Pb}+\mathrm{Pb}$ collisions.

The analytical model of Ref. [9] for

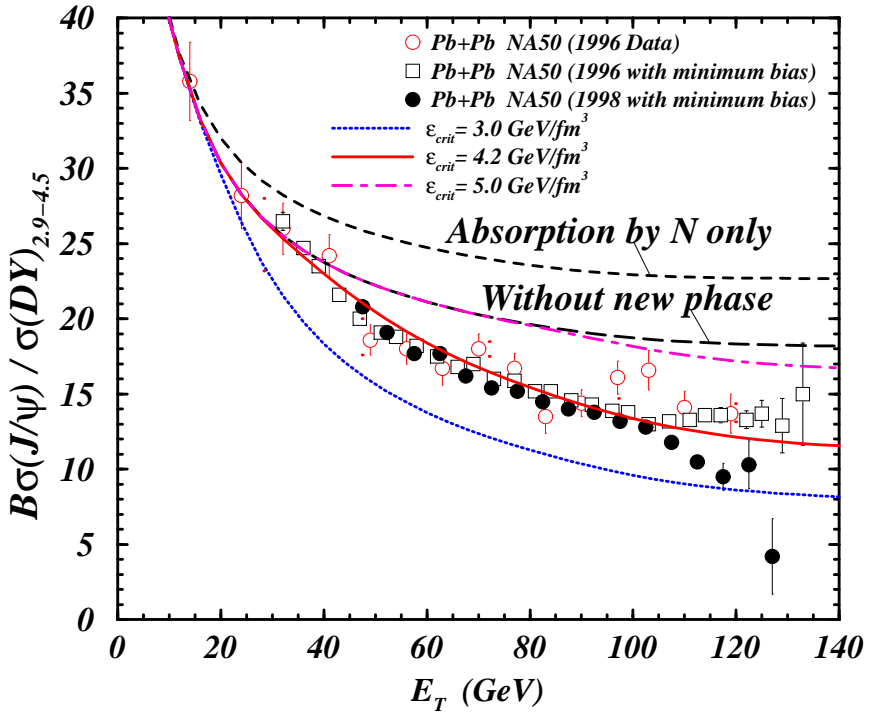

Fig. 5. The ratio of the $J / \psi$ cross section to the Drell-Yan cross section at different values of transverse energy $E_{T}$. The NA50 data are from Ref. [22 24].

$J / \psi$ is useful to provide new insight into the main characteristics of the suppression process. It is desirable to make a more refined calculation to include many improvements in order to see whether the main characteristics may depend on these refinements. The cross section for the dissociation of $J / \psi$ by hadrons should be reliably calculated and their dependence on hadron types and hadron energies included. The population of the $\rho$ meson should also be obtained in a dynamical model where the $\rho$ mesons are both formed by pion collisions and decay back into pions. The effect of the degradation of the energy 
of the colliding nucleons and their subsequent lower hadron production should also be taken into account. These improvements can be included in a Monte Carlo cascade model calculation as shown below.

To obtain reliable $J / \psi$ dissociation cross sections, we first determine the effective quarkgluon potential from hadron masses and use these interactions in the quark-interchange model of Barnes and Swanson [20] to obtain the dissociation cross section. The Barnes and Swanson model has been tested and found to give good agreement with experimental phase shifts and cross sections in many meson-meson reactions.

By using the Barnes and Swanson model, the cross section for the dissociation of $J / \psi$ by a pion is found to be small, with a peak cross section of about $1 \mathrm{mb}$ above the threshold of $640 \mathrm{MeV}$. On the other hand, the cross section for the dissociation by a $\rho$ meson is large because it is above the $D+\bar{D}$ threshold. The cross section decreases rapidly as a function of energy. The dependence of the cross sections on the mass of the $\rho$ meson have also been calculated 28].

Having obtained the dissociation cross sections, we incorporate them in a heavy-ion Monte Carlo cascade program to study the survival probability of a produced $J / \psi$. We use different basic numerical programs in our Monte Carlo cascade calculations [29]. In one of our programs for which we shall report our results, we base our calculations on the MARCO program, which is a multiple collision model with a stopping law to govern the degradation of the energy of nucleons and a particle production law to specify the distribution of produced particles after a nucleon-nucleon collision [30]. The MARCO program has been found to give a good description of the gross features of hadron production [31]. The rescattering of the produced particle is now incorporated to follow the cascade of the hadrons.

In Fig. 6, we plot the $J / \psi$ production cross section as a function of $A^{1 / 3}(A-1) / A+B^{1 / 3}(B-1) / B$, where $A$ and $B$ are the mass numbers of the colliding nuclei. The results from the Monte Carlo cascade calculations are given as the dashed curve. The inclu-

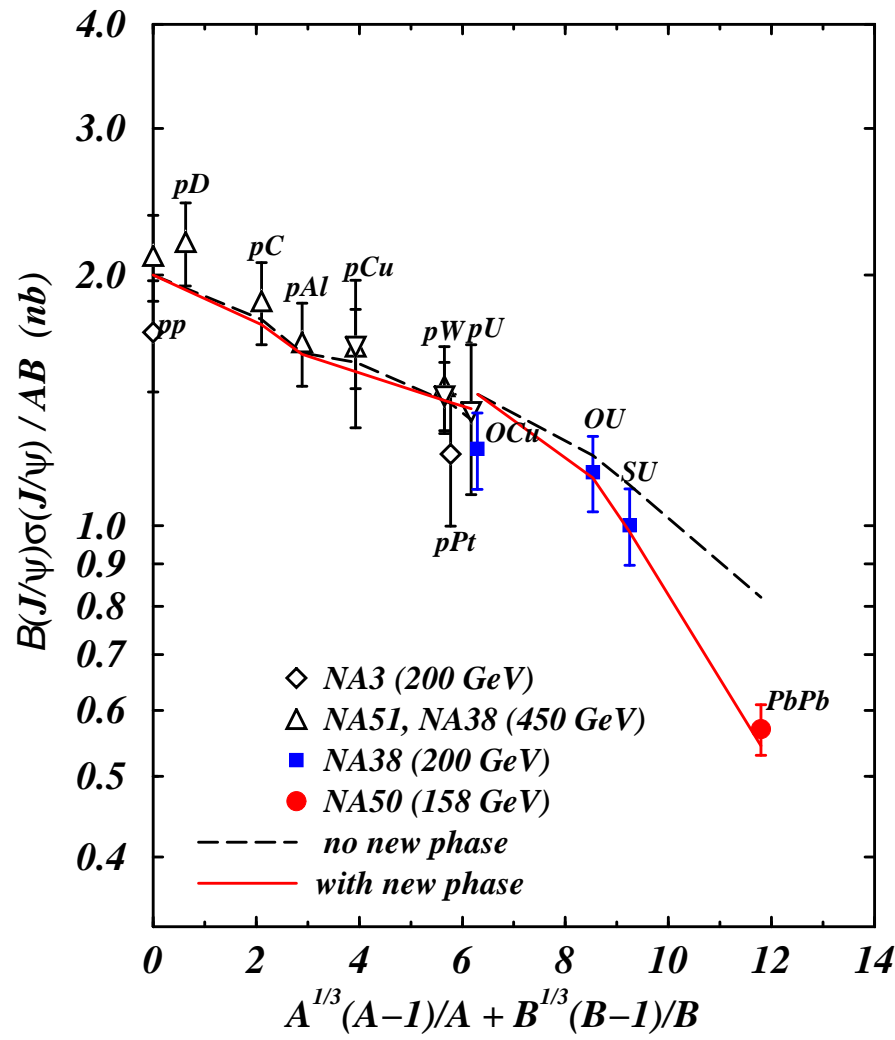

Fig. 6. $J / \psi$ cross section as a function of $A^{1 / 3}(A-1) / A+B^{1 / 3}(B-1) / B$. [11]. sion of the $\rho$ mesons increases the absorption slightly for $\mathrm{S}+\mathrm{U}$ and $\mathrm{Pb}+\mathrm{Pb}$ but does not lead to good agreement with the $\mathrm{Pb}+\mathrm{Pb}$ data. Again, when we postulate that a new phase of $J / \psi$ absorption is produced when the local energy density exceeds a critical value, then we obtain the results as shown in the solid curve. The qualitative features of 
the $J / \psi$ absorption are not changed with a refined calculation for $J / \psi$ production.

\section{CONCLUSION AND SUMMARY}

In high-energy heavy-ion collisions, the occurrence of the quark-gluon plasma will be accompanied by an anomalous increase in pressure, an excess of direct photon production, an excess of strangeness production, and an anomalous $J / \psi$ suppression. We reviewed these signatures and find that they are present in central $\mathrm{Pb}+\mathrm{Pb}$ collisions at $158 \mathrm{~A} \mathrm{GeV}$.

For the indicator of the anomalous increase in pressure, the NA44 data exhibits an anomalous increase in the freeze-out volume as a function of multiplicity. Superimposing on the normal systematic increase of the freeze-out volume due to the increase in the volume of the interacting region may be the additional increase due to the high pressure of the quark-gluon plasma.

For the question of direct photon production, the WA98 Collaboration has measured the direct photon spectrum in central $\mathrm{Pb}+\mathrm{Pb}$ collision, and has found that the photon yield is in excess of the expected yield from nucleon-nucleon hard scattering processes. The excess direct photons can be understood as arising from a quark-gluon plasma with an initial temperature of $300 \mathrm{MeV}$.

For the enhancement of strangeness, the WA97 Collaboration observed a large enhancement in the yield of $\Omega$ hyperons which cannot be explained by RQMD cascade calculations. Such a discrepancy may support the enhancement as originating from the quark-gluon plasma. This evidence needs to be confirmed by calculating the strangeness-raising cross sections with a reliable model.

In the case of $J / \psi$ suppression, the anomalous suppression in central $\mathrm{Pb}+\mathrm{Pb}$ collisions cannot be explained by the absorption by hadrons. This was demonstrated in a simple analytical model and in a Monte Carlo cascade model using dissociation cross sections obtained from the model of Barnes and Swanson. On the other hand, a critical energy density model can explain the anomalous $J / \psi$ suppression, suggesting the production of the quark-gluon plasma in central $\mathrm{Pb}+\mathrm{Pb}$ collisions at $158 \mathrm{~A} \mathrm{GeV}$.

In conclusion, our re-assessment of these signatures provides additional support to indicate that, in line with the summary of Heinz and Jacobs [1], the experimental data appear to be consistent with the occurrence of the quark-gluon plasma in central $\mathrm{Pb}+\mathrm{Pb}$ collisions at $158 \mathrm{~A} \mathrm{GeV}$.

The author would like to thank Drs. T. Awes, C. M. Ko, M. Murray, and N. Xu for helpful discussions. The author would like to thank Dr. H. Boggild for his permission to show the data of the NA44 Collaboration (Fig. 3). He would also like to thank his collaborators, Drs. T. Barnes, E. Swanson, S. Sorensen, and B. H. Sa, for their collaboration to study $J / \psi$ dissociation cross sections and $J / \psi$ suppression.

\section{REFERENCES}

1. U. Heinz and M. Jacobs, nucl-th/0002042.

2. E. L. Feinberg, Nuo. Cim., 34 (1976) 391; E. Shuryak, Phys. Lett. B 78 (1978) 150.

3. J. Rafelski, Phys. Rep. 88 (1982) 331.

4. T. Matsui and H. Satz, Phys. Lett. B178 (1986) 416. 
5. P. Braun-Munzinger, these Proceedings.

6. C. Y. Wong, Introduction to High-Energy Heavy-Ion Collisions, World Scientific Publishing Company, 1994.

7. JLQCD Collaboration, S. Aoki et al., Nucl. Phys. Proc. Suppl. 73 (1999) 459, heplat/9809102.

8. F. Karsch, Talk presented at Strong and Electroweak Matter '98, Copenhagen, December 1998, hep-lat/9903031.

9. C. Y. Wong, Nucl. Phys. A610 (1996) 434c; C. Y. Wong, Nucl. Phys. A630 (1998) 487; C. Y. Wong, hep-ph/9809497, in Proceedings of Workshop on Charmonium Production in Relativistic Nuclear Collisions, Seattle, May 18-22 1998.

10. J. D. Bjorken, D27 (1983) 140.

11. NA44 Collaboration, I. G. Bearden et al., to be published in Eur. Physics Journal.

12. K. Kajantie, J. Kapusta, L. McLerran, and A. Mekjian, Phys. Rev. D34 (1986) 2746.

13. J. Kapusta, P. Lichard, and D. Seibert, Phys. Rev. D44 (1991) 2774.

14. WA98 Collaboration, M. M. Aggarwal et al., Preprint "Direct Photon Production in $158 \mathrm{~A} \mathrm{GeV}{ }^{208} \mathrm{~Pb}+{ }^{208} \mathrm{~Pb}$ Collisions", to be published.

15. C. Y. Wong and H. Wang, Phys. Rev. C58 (1998) 376.

16. WA97 Collaboration, E. Andersen et al., Phys. Lett. B449 (1999) 401.

17. G. Odyniec, these Proceedings.

18. H. Sorge, Phys. Rev. C52 (1995) 3291.

19. WA97 Collaboration, F. Antinori et al., Eur. Phys. J. C11 (1999) 79.

20. T. Barnes and E. S. Swanson, Phys. Rev. D46, 131 (1992).

21. C. M. Ko, private communications.

22. M. Gonin, NA50 Collaboration, Nucl. Phys. A610 (1996) 404c.

23. A. Romana et al., NA50 Collaboration, in Proceedings of the XXXIII Recontres de Moriond, Les Arcs, France, 21-28 March, 1998.

24. NA50 Collaboration, M. C. Abreu et al., Phys. Lett. B477 (2000) 28.

25. D. Kharzeev, Nucl. Phys. A610 (1996) 418c.

26. J.-P. Blazoit and J.-Y. Ollitrault, Nucl. Phys. A610 (1996) 452c.

27. S. Gavin and R. Vogt, Nucl. Phys. A 610 (1996) 442c; A. Capella et al. Phys. Lett. B393 (1997) 431; W. Cassing and C. M. Ko, Phys. Lett. B396 (1997) 39; Sa BenHao et al., J. Phys. G25 (1999) 1123; E. Shuryak and D. Teaney, Phys. Lett. B 430 (1998) 37; N. Hammon et al., Phys. Rev. C 59 (1999) 2744; D. E. Kahana and S. H. Kahana, Phys. Rev. C 59 (1999) 1651; C. Gale, S. Jeon, and J. Kapusta, Phys. Lett. B 459 (1999) 455; M. Nardi and H. Satz, Phys. Lett. B442 (1998) 14; H. Satz, hep-ph/0007609.

28. C.Y. Wong, E. S. Swanson, and T. Barnes, hep-ph/9912431, in press in Phys. Rev. C; C.Y. Wong, E. S. Swanson, and T. Barnes, nucl-th/0002034, Proceedings of the 28th International Workshop on Gross Properties of Nuclei and Nuclear Excitation, Hirschegg, Austria, 2000; T. Barnes, E. S. Swanson, and C.Y. Wong, nucl-th/0006012, Proccedings of 5th International Workshop on Heavy Quark Physics, Dubna, 2000.

29. C. Y. Wong, S. Sorensen, and B. H. Sa, to be published.

30. C.Y. Wong and Z. D. Lu, Phys. Rev. C39 (1989) 2606.

31. J.Y. Zhang, X. He, C. C. Shih, S. Sorensen, and C. Y. Wong, Phys. Rev. C46 (1992) 748. 